\title{
Heterogeneous Microcirculation in the Rat Small Intestine during Hemorrhagic Shock: Quantification of the Effects of Hypertonic-Hyperoncotic Resuscitation
}

\author{
K. Vajda Andrea Szabó $^{b} \quad$ M. Boros ${ }^{b}$ \\ ${ }^{a}$ Department of Surgery, County Teaching Hospital, Kecskemét, and ${ }^{b}$ Institute of Surgical Research, University of \\ Szeged, Szeged, Hungary
}

\section{Key Words}

Hypertonic/hyperoncotic solution - Mucosa - Muscle

layer - Microcirculatory heterogeneity

\begin{abstract}
Purpose: In the event of a spatial or temporal microvascular perfusion heterogeneity conventional methods are often inadequate to describe the microcirculatory changes. Our aim was to use a new formula to characterize and compare the microcirculatory reactions in the mucosa and longitudinal muscle of the rat small intestine in response to hypertonic/hyperoncotic and normotonic resuscitation strategies. Methods: Intravital videomicroscopy with an orthogonal polarization spectral (OPS) imaging technique was utilized. Microcirculatory variables were recorded during hemorrhagic shock (HS; $50 \mathrm{~mm} \mathrm{Hg}$ mean arterial pressure for $60 \mathrm{~min}$ ) and fluid replacement with $0.9 \%$ saline or with $7.2 \%$ saline containing 10\% hydroxyethylstarch 200/0.5 (Osmohes; $4 \mathrm{ml} /$ $\mathrm{kg}$ ). Due to the temporal perfusion variability, microcirculatory changes were described using the calculation of the average red blood cell velocity (A-RBCV), while the spatial changes were calculated as a function of the size of the perfused capillary network. Results: During HS and the late phase of resuscitation, perfusion was char-
\end{abstract}

\section{KARGER}

Fax +41613061234

E-Mail karger@karger.ch www. karger.com

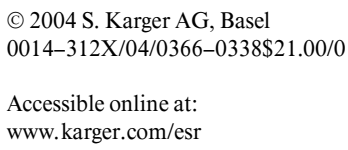

acterized by capillary flow motion (i.e. variability in time) in the villi, and by spatial flow heterogeneity in the longitudinal muscle layer. The approximately $40 \%$ decrease in the calculated villus A-RBCV during HS was only partially affected by $0.9 \%$ saline, whereas Osmohes completely restored A-RBCV by increasing both the red blood cell velocity and the duration of high-flow periods at the onset of resuscitation in the villi. The approximately $60 \%$ reduction in $\mathrm{A}-\mathrm{RBCV}$ in the muscle layer during $\mathrm{HS}$ was not followed by an appreciable recovery in either group, but Osmohes significantly increased A-RBCV in the late resuscitation phase. Conclusions: The hypertonic/hyperoncotic solution induces a considerable microcirculatory improvement in two distinct layers of the small intestine after HS. This positive effect is related to the amelioration of the intestinal microcirculatory heterogeneity.

Copyright $@ 2004$ S. Karger AG, Basel

\section{Introduction}

Severe hemorrhage causes a disproportionate compensatory reaction in the mesenteric circulation which leads to persistent gastrointestinal hypoperfusion [1]. Since mucosal ischemia predisposes to the influx of luminal foreign
Andrea Szabó, MD, PhD

Institute of Surgical Research, University of Szeged

Pécsi u. 4

HU-6720 Szeged (Hungary)

Tel. +3662 545103, Fax +36 62 545743, E-Mail sza@expsur.szote.u-szeged.hu 
material [2], a prolonged splanchnic flow reduction could promote the development of septic complications and multiple organ failure [3, 4]. A major aim of primary volume resuscitation is to reverse maldistribution of the blood flow in the macro- and microcirculation, but the microcirculatory derangement may be sustained in spite of volume therapy and improved macrohemodynamics $[1,5-7]$. Although the significance of a compromised microcirculation is clearly recognized, the characterization of dynamically varying microcirculatory changes can be very difficult. We recently proposed a new method which allows a comparison of timewise and spatial perfusion heterogeneity [8]. The results showed that despite the perfusion heterogeneity, microcirculatory changes can be conveniently described by calculation of the timewise variability of the capillary red blood cell velocity (RBCV).

Hypertonic and/or hyperoncotic solutions have been demonstrated to bring about rapid improvements in both the macro- and the microhemodynamics of splanchnic organs [6, 9-11]. The rationale of low-volume fluid resuscitation provided by these solutions is to avoid a rapid increase in blood pressure, which would cause further bleeding before the appropriate surgical control becomes available. It appears that the beneficial effects are related to a number of mechanisms including the redistribution of fluid from the interstitium to the intravascular space [12]; an increase in cardiac output without a rise in mean arterial pressure (MAP) [13]; a direct relaxing action on the vascular smooth muscle $[7,14]$; a positive inotropic effect on heart contractility $[15,16]$; an improvement in the blood fluidity by hemodilution [17]; metabolic effects [13], and a reduction in the leukocyte-endothelial interactions [18-20]. The hypertonic/hyperoncotic solution of $10 \%$ dextran 60 in $7.2 \%$ saline was proven to be most effective in improving organ perfusion during traumatichemorrhagic hypotension [10].

The primary aim of our present study was to observe microcirculatory consequences of hypovolemia in different anatomical layers of the small intestine in a rat model of hemorrhagic shock (HS). Secondly, we compared the effectiveness of hypertonic/hyperoncotic colloid and normotonic crystalloid resuscitation by using our previously proposed formula [8]. The results demonstrate that the microcirculatory consequences of fluid therapy are not necessarily related to macrohemodynamic changes, and a detailed analysis should be performed to quantify the intramural microcirculatory flow pattern changes and the efficacy of different resuscitation strategies.

\section{Materials and Methods}

The experiments were performed in accordance with the NIH Guidelines (Guide for the Care and Use of Laboratory Animals) and the study was approved in advance by the Animal Welfare Committee of the University of Szeged.

\section{Surgical Preparation}

Male Wistar rats (average weight $300 \pm 20 \mathrm{~g}$ ) housed in an environmentally controlled room with a 12-hour light-dark cycle were deprived of food, but not water, for $12 \mathrm{~h}$ prior to the experiments. The animals were anesthetized with intraperitoneal sodium pentobarbital $(45 \mathrm{mg} / \mathrm{kg}$, small supplementary doses were intravenously administered later when necessary) and placed in a supine position on a core temperature-controlled heating table $\left(38^{\circ} \mathrm{C}\right)$. The left jugular vein and the right carotid artery were cannulated for fluid and drug administration and for the measurement of MAP, respectively. Small supplementary doses of pentobarbital were administered intravenously when necessary. The trachea was cannulated to facilitate respiration and the animals received a continuous infusion of isotonic saline $(10 \mathrm{ml} / \mathrm{kg} / \mathrm{h})$. A midline laparotomy was performed and two ileal segments were selected and exteriorized, their neurovascular supply being maintained intact. An ileal loop was placed on a specially designed stage, covered with a microscopic glass cover slip and used for visualization of the longitudinal muscle layer from the serosal orientation. A small antimesenteric enterotomy was performed by means of electrocautery on the second segment, to expose the mucosal surface and visualize the mucosal villi. The lumen of this segment was flushed with warm saline and placed on another pedestal, and the mucosal surface was also covered with a cover slip. The rest of the abdominal cavity was covered with gauze sponges soaked in saline and Saran wrap in order to prevent fluid and heat loss.

\section{Microcirculatory Measurements}

The microcirculation of the mucosal villi and the longitudinal muscle was continuously visualized by intravital videomicroscopy with the OPS imaging technique (CYTOSCAN A/R, Cytometrics, $\mathrm{Pa}$., USA). The OPS technique utilizes epi-illumination with linearly polarized light at $548 \mathrm{~nm}$ (which is the isobestic point of oxy- and deoxyhemoglobin) to visualize hemoglobin-containing structures without the additional use of a fluorochrome. Since polarization is preserved in reflection, only photons scattered from relatively deep in the tissues (approx. $200 \mu \mathrm{m}$ ) contribute to the images. In this way, a virtual light source is created in the tissues such that the depolarized scattered light effectively back-illuminates the structures in the foreground. The device thereby provides an optimal imaging of hemoglobin-containing microvascular structures for intravital microscopy at a chosen focus level [21]. Videomicroscopic images were recorded intermittently with an S-VHS videorecorder (Panasonic AG-MD 830). Quantitative assessment of the microcirculatory parameters was performed off-line by frame-to-frame analysis of the videotaped images. The functional capillary density (FCD), the length of the red cell perfused capillaries per observation area $\left(\mathrm{cm} / \mathrm{cm}^{2}\right)$ of the villi and the capillary RBCV $(\mu \mathrm{m} / \mathrm{s})$ were determined in 3 separate fields in both layers (at least 10 measurements were made at each time point) by means of a computer-assisted image analysis system (IVM Pictron, Budapest, Hungary). 


\section{Experimental Protocol}

Surgery was followed by a 15 -min stabilization period, and the baseline variables were then recorded. The animals were randomly assigned into one of 3 groups. In group $1(n=6)$, sham operation was performed, and the microcirculatory variables were recorded for $180 \mathrm{~min}$ to exclude any changes relating solely to the anesthesia and surgery. In group $2(n=6)$, blood was withdrawn from the femoral artery into a heparinized syringe until the MAP reached $50 \mathrm{~mm} \mathrm{Hg}$, and blood was then additionally withdrawn or retransfused to maintain the set MAP value for $60 \mathrm{~min}$. Fluid replacement with $0.9 \%$ saline $(200 \%$ of the shed blood volume, followed by $15 \mathrm{ml} / \mathrm{kg} / \mathrm{h}$ lactated Ringer's solution) was started after hemorrhage. In group 3 ( $\mathrm{n}=$ 5), the fluid replacement was conducted with $7.2 \%$ saline containing 10\% HES 200/0.5 (Osmohes ${ }^{\circledR}$, Fresenius Kabi GmbH, Graz, Austria; $4 \mathrm{ml} / \mathrm{kg}$ ) over $4 \mathrm{~min}$, followed by $15 \mathrm{ml} / \mathrm{kg} / \mathrm{h}$ lactated Ringer's solution. Both HS groups were observed for $120 \mathrm{~min}$ in the resuscitation period.

\section{Statistical Analysis}

In all figures, data are expressed as means \pm SDs. Data analysis was performed with a statistical software package (SigmaStat 1.0 for Windows, Jandel Scientific, Erkrath, Germany). Changes in variables within groups were analyzed by two-way ANOVA tests, followed by the Bonferroni test. Differences between groups were evaluated by means of Student's unpaired t test. Coefficient of variation was calculated as $\mathrm{SD} /$ mean. $\mathrm{p}<0.05$ was considered statistically significant.

\section{Results}

HS was induced by withdrawing similar volumes of blood in both experimental groups $(6.7 \pm 0.9$ and $7.2 \pm$ $0.8 \mathrm{ml}$ with $0.9 \%$ saline and Osmohes resuscitation, respectively).

During resuscitation, the MAP did not reach the baseline in the $0.9 \%$ saline-resuscitated group (baseline: 105 $\pm 6 \mathrm{~mm} \mathrm{Hg}, \mathrm{t}=15 \mathrm{~min}: 86 \pm 10 \mathrm{~mm} \mathrm{Hg}, \mathrm{p}<0.001)$, but it was rapidly restored and maintained for $60 \mathrm{~min}$ after Osmohes (baseline: $96 \pm 8 \mathrm{~mm} \mathrm{Hg}, \mathrm{t}=15 \mathrm{~min}$ : $106 \pm$ $20 \mathrm{~mm} \mathrm{Hg}, \mathrm{t}=60 \mathrm{~min}: 90 \pm 22 \mathrm{~mm} \mathrm{Hg}$ ) which was followed by a gradual decrease in both groups. As a result, $3 / 6$ of the $0.9 \%$ saline-treated and $3 / 5$ animals of the Osmohes-treated animals survived the 120-min resuscitation period (survival times of $89 \pm 35$ and $94 \pm 36 \mathrm{~min}$, respectively; $p=0.83$ ).

In the sham-operated animals, the microhemodynamic parameters did not change significantly over time and the microcirculatory perfusion was always continuous in both structures (the RBCV was approx. $600 \mu \mathrm{m} / \mathrm{s}$ in the villi, and $620 \mu \mathrm{m} / \mathrm{s}$ in the muscle layer, respectively).

During HS and the late phase of resuscitation, the perfusion was characterized by capillary flowmotion (i.e. variability in time) in the villi, and by spatial flow heterogeneity in the longitudinal muscle layer. Accordingly, the simple arithmetic average of RBCV could not be used to calculate the average RBCV (A-RBCV) or to compare the continuous perfusion with the oscillatory flow pattern in the villi (problem I), nor homogeneous and heterogeneous perfusion in the muscle (problem II). Specifically, hemorrhage induced a time-dependent fluctuation in flow in the villus capillaries, with an alternating sequence of high $(\sim 380-440 \mu \mathrm{m} / \mathrm{s})$ and low $(\sim 120-140 \mu \mathrm{m} / \mathrm{s})$ velocity. The flowmotion was not confined to the capillaries, but could be observed in all the microvascular structures of the villi (capillaries, subepithelial venules and central arterioles). The flow pattern displayed a square wave character, the initiation and cessation of flow were abrupt; hence, the durations of the different periods could be accurately measured [see 22 , for schematic depiction of data analysis]. For these reasons, we characterized the velocities according to their magnitude and duration in the villi and calculated A-RBCV from the sum of the individual RBCV values (equations 1 and 2) and used the corresponding durations of periodicity as weighting factors (eq. 1).

$$
\mathrm{A}-\mathrm{RBCV}=\frac{\mathrm{V}_{1} \mathrm{~T}_{1}+v_{1} t_{1}+\mathrm{V}_{2} \mathrm{~T}_{2}+v_{2} t_{2}+\cdots \mathrm{V}_{n} \mathrm{~T}_{n}+v_{n} t_{n}}{\mathrm{~T}_{1}+t_{1}+\mathrm{T}_{2}+t_{2}+\cdots \mathrm{T}_{n}+t_{n}}
$$

In this formula, $\mathrm{V}_{\mathrm{n}}$ and $\mathrm{T}_{\mathrm{n}}$ are the RBCVs and the relative durations referring to the high-flow periods, while $v_{n}$ and $t_{n}$ are the RBCVs and relative durations referring to the low-flow periods.

Accordingly, approximately $40 \%$ in A-RBCV was demonstrated during $\mathrm{HS}$ in the villi. The hypoperfusion in the villi was only partially affected by $0.9 \%$ saline, since A-RBCV did not reach the baseline values during resuscitation (fig. 1). However, Osmohes completely restored A-RBCV by increasing both RBCV values and the duration of high-flow periods at the onset of resuscitation in the villi without influencing the late resuscitation phase.

Severe perfusion heterogeneity was observed in the longitudinal muscle as well. Here, capillaries with complete stasis were not identified, and therefore the FCD did not change significantly (data not shown). Nonetheless, certain capillaries were characterized by a continuous flow of erythrocytes with very low RBCV (ranging between 50 and $180 \mu \mathrm{m} / \mathrm{s}$ ), and others with relatively high velocities (ranging between 310 and $600 \mu \mathrm{m} / \mathrm{s}$ ). The highand low-flow capillaries could be readily discerned and their lengths measured. Again, A-RBCV was calculated from the sum of the individual velocity values and the 


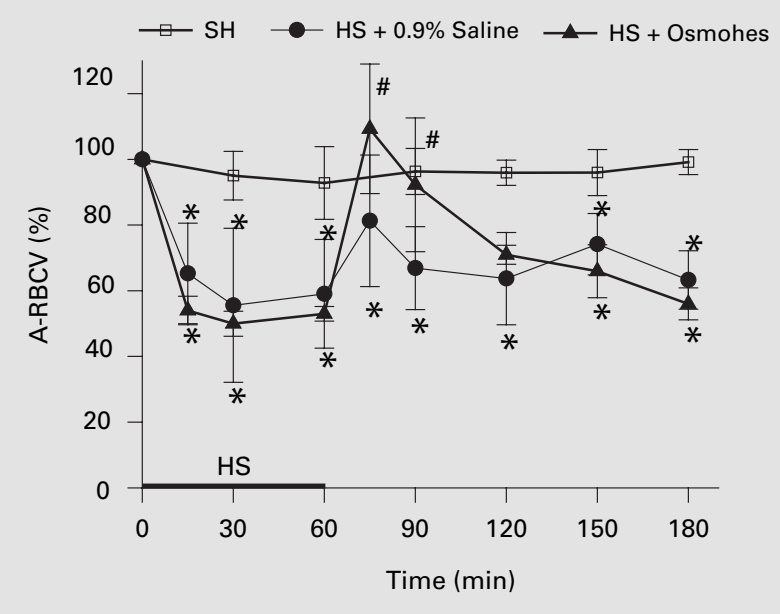

Fig. 1. Changes in time of average red blood cell velocity (A-RBCV) in the capillaries of the mucosal villi during sham operation $(\mathrm{SH})$, or during 60 -min hemorrhagic shock (HS) followed by $0.9 \%$ saline resuscitation or Osmohes $(4 \mathrm{ml} / \mathrm{kg})$ treatment. Data are means $\pm \mathrm{SD}$. $* \mathrm{p}<0.01$ vs. baseline, $\# \mathrm{p}<0.05$ vs. saline.

relative length of the capillaries was used as weighting factor (eq. 2).

$$
\mathrm{A}-\mathrm{RBCV}=\frac{\mathrm{VL}+v l}{\mathrm{~L}+l}
$$

where $\mathrm{V}$ and $\mathrm{L}$ are the average $\mathrm{RBCV}$ referring to highflow areas and the relative length of the capillaries in the corresponding area, respectively, while $v$ and $l$ are the average $\mathrm{RBCV}$ and the corresponding relative length of the capillaries referring to the low-flow areas, respectively.

Accordingly, A-RBCV decreased by approximately $60 \%$ in muscle layer during HS. At resuscitation onset, A-RBCV reached approximately $65 \%$ of the baseline with $0.9 \%$ saline, but was followed by a further derangement as marked by an A-RBCV characteristic of the hypovolemic phase (fig. 2). The resuscitation onset with Osmohes was characterized by a similar degree of microvascular perfusion recovery in the muscle layer, but A-RBCV was later significantly higher as compared with that observed on $0.9 \%$ saline treatment.

In order to characterize the heterogeneity itself, we expressed the changes in heterogeneity in time and space by calculating the coefficient of variation of RBCV values. HS was associated with a marked increase in perfusion heterogeneity as evidenced by the increase in the coefficient of variation of RBCV from approx. 0.08-0.09

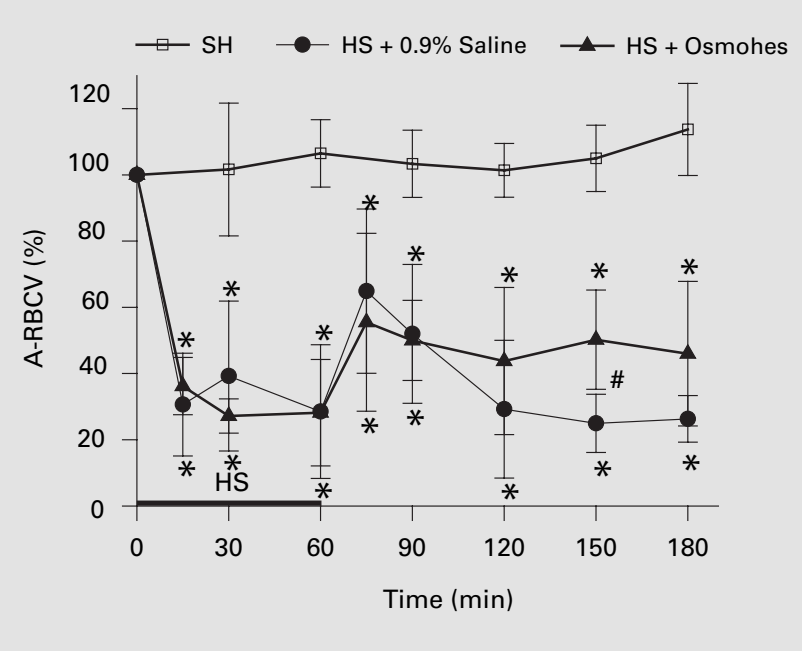

Fig. 2. Changes in time of average red blood cell velocity (A-RBCV) in the capillaries of the longitudinal muscle layer during sham operation $(\mathrm{SH})$, or during 60 -min hemorrhagic shock (HS) followed by $0.9 \%$ saline resuscitation or Osmohes $(4 \mathrm{ml} / \mathrm{kg})$ treatment. Data are means \pm SD. ${ }^{*} \mathrm{p}<0.01$ vs. baseline, $\# \mathrm{p}<0.05$ vs. saline.

baseline to $0.38-0.40$ and $0.32-0.62$ in the villi and muscle, respectively.

Along with the reestablishment of continuous flow conditions at the onset of reperfusion, the early phase of resuscitation resulted in an amelioration of perfusion heterogeneity in the villi. The coefficient of variation of RBCV decreased to near baseline values in response to both resuscitation strategies (to $0.23 \pm 0.20$ and $0.09 \pm$ 0.01 after saline and Osmohes treatment, respectively). This was followed by a gradual microcirculatory deterioration after physiological saline treatment since the coefficient of variation of $\mathrm{RBCV}$ was again significantly higher than in the sham-operated animals or at baseline. In the Osmohes-treated group the amelioration was maintained until the 30th min of resuscitation $(0.18 \pm 0.12$ and 0.57 \pm 0.13 in Osmohes vs. saline-treated group, respectively; $\mathrm{p}<0.05)$.

In the muscle layer, however, no significant improvement was observed in this parameter during the resuscitation. The coefficient of variation of RBCV was persistently and significantly higher in both hemorrhaged groups (0.42-0.56 and 0.41-0.48 after saline and Osmohes treatment, respectively) as compared to shamoperated animals.

Villus FCD was determined during the high-flow phases of oscillation. With this restriction, HS was not

Timewise and Spatial Flow Heterogeneity 


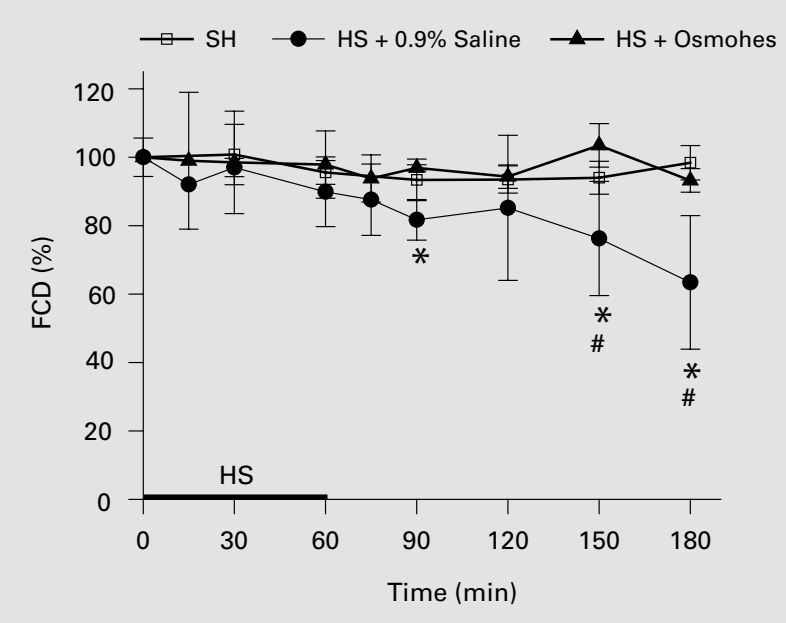

Fig. 3. Changes in time of functional capillary density (FCD) of the mucosal villi during sham operation $(\mathrm{SH})$, or during 60 -min hemorrhagic shock (HS) followed by $0.9 \%$ saline resuscitation or Osmohes $(4 \mathrm{ml} / \mathrm{kg})$ treatment. Data are means $\pm \mathrm{SD} .{ }^{*} \mathrm{p}<0.05$ vs. baseline, $\# \mathrm{p}<0.05$ vs. sham.

associated with significant changes, but the FCD gradually decreased (by 30\%) in the late phase of resuscitation with saline, while it was kept on the baseline level in the Osmohes-resuscitated group (fig. 3).

\section{Discussion}

Restoration of the microcirculation is a good indicator of the effectiveness of resuscitation strategies, but the commonly used parameters do not allow a comparison of the dynamically altering microcirculatory changes [2326]. In our study, HS and the late phase of resuscitation were characterized by capillary flowmotion (i.e. variability in time) in the villi, and by perfusion heterogeneity (variability in space) in the longitudinal muscle layer. Therefore, the simple arithmetic average of RBCV could not be used to compare the continuous perfusion with the oscillatory flow pattern (problem I), or homogeneous and heterogeneous perfusion in a given tissue layer (problem II). For these reasons, we proposed the use of the weighted arithmetic average of RBCV (A-RBCV), which is calculated from the sum of the individual RBCVs and different weighting factors. In this way, factors of variability such as the duration of periodicity or the relative length of wellperfused capillaries are considered and included in the calculations. Hence, a microcirculatory improvement could be characterized relatively easily. Nevertheless, these parameters could be quantified only by means of a detailed microcirculatory analysis.

With respect to the timewise heterogeneity, the deteriorating intestinal circulation was accompanied by periodic fluctuations in capillary blood flow, generally termed flowmotion [24-26]. Since this microvascular oscillatory phenomenon was not observed under control conditions, its appearance can be regarded as a manifestation of microcirculatory impairment and increased tissue perfusion heterogeneity at any later stage of the experiments. Accordingly, the short-term beneficial effect of fluid resuscitation provided by Osmohes is related not only to the temporary restoration of near-normal microcirculatory velocities, but also to the re-established continuous flow conditions or to the increased length of the high-flow phases during flowmotion. This further underlines the importance of including the duration of distinct flow periods in the data analysis. It is also noteworthy that the microcirculatory improvement provided by Osmohes is only temporary when it is not followed by resuscitation with higher volumes.

It is proposed that flowmotion develops on the basis of vasomotion [27] derived from their simultaneous occurrence, as seen in the skeletal muscle and pancreas [24, 28]. Until recently, the presence of physiological vasomotion and flowmotion was a matter of debate [28]. Interestingly, in earlier studies it was not the enhancement, but rather the abolishment of spontaneous arteriolar vasomotion that was attributed to the precapillary constriction [29, 30]. It should be noted that the standard frame-to-frame analysis applied here could not detect small RBCV amplitude differences which may be present during physiological capillary flowmotion. Hypovolemic hypotension, however, resulted in a sufficient degree of increase in flowmotion, i.e. it caused such great low/high-value differences in RBCV that it could easily be traced during data analysis.

The cause of flowmotion is unclear, but a new balance between vasoconstrictor and vasodilator forces can be presumed behind this phenomenon. Flow oscillation could be a protective mechanism since it has been shown to improve the efficacy of tissue oxygenation under lowflow conditions [31]. It is conceivable that endogenous catecholamines contribute to local flow pattern regulation, because HS is characterized by an intense neuroendocrine response and stress hormone release [32], while norepinephrine induces periodic, intracellular calciummediated changes in the membrane potential of vascular 
smooth muscle cells in vitro [33], and increases flow oscillations in the brain [25]. It is important that the oscillatory phenomenon was not confined to the capillaries: the cessation of flow started in the central arteriole. Hence, the vasoconstriction did not arise from precapillary sphincters, but probably from 3A-type upstream arterioles (according to the description of Bohlen and Gore [34]). The synchronized flow patterns of adjoining villi support this hypothesis. Hypertonic fluids have been shown to exert a relaxing action on vascular smooth muscle or to reduce the resistance of the microvascular network directly. As such, a normalization of 1A-type arteriolar diameters and a decrease in vascular resistance have been demonstrated in response to hypertonic solutions in other models [35]. Nonetheless, elucidation of the mechanism involved in the improvement provided in the villus microcirculation by the hypertonic/hyperoncotic solution applied here warrants further investigations.

With regard to the spatial perfusion heterogeneity, it is important to note that the muscle microcirculation deteriorated to a greater extent than that of the mucosa (see A-RBCV data), indicating a redistribution of the microvascular blood flow from the muscle toward the mucosa. Another feature is that a conventional measure of microvascular injury (i.e. FCD) did not reveal the decrease in efficacy of the tissue perfusion in the longitudinal muscle layer. Specifically, the proportion of capillaries exhibiting stasis (generally described as a decrease in FCD) did not increase during reperfusion, but rather the number of microvessels characterized by lower velocities increased. The new approach used here to estimate the decreased efficacy of capillary perfusion sensitively described the microcirculatory changes, and provided a basis for comparison despite the increased heterogeneity of capillary flow within the muscle layer. The analysis also demonstrated that the hyperoncotic solution ameliorated the deterioration in muscle perfusion in the late resuscitation phase.

The pathophysiological background of increased spatial perfusion heterogeneity additionally remains to be characterized. It is reasonable to assume that at least two distinct mechanisms play roles in mediating the in-layer and between-layer microcirculatory disturbances during HS: vasoconstriction and endothelial cell swelling. Our observations lead to the suspicion of arteriolar vasoconstriction in the background of the intramural flow redistribution at the level of the mucosa and muscle border. This has also been suggested by other authors, the role of type $3 \mathrm{~A}$ arterioles of the intestinal wall being discussed in the background of a similar phenomenon in endotoxemia
[36]. In our study, highly severe microcirculatory disturbances such as decreases in FCD were not seen in the muscle layer, but it seems reasonable to assume that changes in FCD are mediated by direct endothelial damage in the capillary network. The swelling of endothelial cells can cause narrowing and obstruction of the capillary lumen. The resultant elevation in the hydraulic resistance directs the blood flow to microcirculatory segments of lower resistance. The consequent perfusion heterogeneity is regarded as a hallmark of the microcirculation in shock termed the 'no-reflow phenomenon' [37]. In line with these assumptions, it is well known that the microcirculatory improvement provided by hyperoncotic solutions can be characterized by an 'internal transfusion phenomenon' originating from a shift of water from erythrocytes and endothelial cells first, and from the interstitium and parenchymal tissue cells later, resulting in an increase in the intravascular volume $[12,26]$. Indeed, Osmohes prevented the loss of FCD in villi during the late stages of resuscitation when muscle microcirculation was also moderately improved. Therefore, our observations indicate that the hyperoncotic resuscitation probably influences both vasoconstrictor forces and endothelial injury.

In conclusion, the present study demonstrates that HS is accompanied by characteristic temporal and spatial heterogeneity, which cannot be precisely quantified by means of conventional data analysis. We propose an approach which takes into account the major factors of heterogeneity and allows a comparison of different flow patterns. The analysis revealed that hypertonic/hyperoncotic resuscitation after HS leads not only to changes in standard microcirculatory parameters (e.g. RBCV and FCD), but also influences the factors of perfusion heterogeneity. The results showed that the microcirculatory improvement in two distinct layers of the small intestine after hypertonic/hyperoncotic volume replacement is related to the amelioration of the microcirculatory heterogeneity.

\section{Acknowledgments}

This work was supported by research grants from the Hungarian Scientific Health Council (ETT 686/2001 and ETT 162/2002) and the Scientific Research Fund (OTKA T037392). A.S. is a Bolyai Fellow of the Hungarian Academy of Sciences. 


\section{References}

1 Scannell G, Clark L, Waxman K: Regional flow during experimental hemorrhage and crystalloid resuscitation: Persistence of low flow to the splanchnic organs. Resuscitation 1992;23:217225.

2 Wells C, Maddaus M, Simmons R: Bacterial translocation; in Marston A, Bulkley G, Fiddian-Green R, Haglund U (eds): Splanchnic Ischemia and Multiple Organ Failure. St Louis, Mosby, 1989, vol 17, pp 195-203.

-3 Deitch EA: The role of intestinal barrier failure and bacterial translocation in the development of systemic infection and multiple organ failure. Arch Surg 1990;125:403-404.

-4 Faist E, Baue AE, Dittmer H, Heberer G: Multiple organ failure in polytrauma patients. $\mathrm{J}$ Trauma 1983;23:775-787

$>5$ Wang P, Hauptman JG, Chaudry IH: Hemorrhage produces depression in microvascular blood flow which persists despite fluid resuscitation. Circ Shock 1990;32:307-318.

6 Messmer K, Kreimeier U: Microcirculatory therapy in shock. Resuscitation 1989;18:S51S61.

7 Scalia S, Burton H, Van Wylen D, Steinberg S, Hoffman A, Roche F, Flint L: Persistent arteriolar constriction in microcirculation of the terminal ileum following moderate hemorrhagic hypovolemia and volume restoration. J Trauma 1990;30:713-718.

8 Vajda K, Szabó A, Kucsa K, Suki B, Boros M: Microcirculatory heterogeneity in the rat small intestine during compromised flow conditions. Microcirculation 2004; in press.

$\checkmark$ Behrman SW, Fabian TC, Kudsk KA, Proctor KG: Microcirculatory flow changes after initial resuscitation of hemorrhagic shock with $7.5 \%$ hypertonic saline $/ 6 \%$ dextran 70 . J Trauma 1991;31:589-598.

- 10 Kreimeier U, Brückner UB, Niemczyk S, Messmer K: Hyperosmotic saline dextran for resuscitation from traumatic-hemorrhagic hypotension: effect on regional blood flow. Circ Shock 1990;32:83-99.

11 Vollmar B, Lang G, Menger MD, Messmer K Hypertonic hydroxyethyl starch restores hepatic microvascular perfusion in hemorrhagic shock. Am J Physiol 1994;266:H1927-H1934.

12 Mazzoni MC, Borgström P, Arfors KE, Intaglietta M: Dynamic fluid redistribution in hyperosmotic resuscitation of hypovolemic hemorrhage. Am J Physiol 1988;255:H629-H637.
13 Dubick MA, Wade CE: A review of the efficacy and safety of $7.5 \% \mathrm{NaCl} / 6 \%$ dextran 70 in experimental animals and in humans. $\mathbf{J}$ Trauma 1994;36:323-330.

14 Gazitua S, Scott JB, Swindall B, Haddy FJ: Resistance responses to local changes in plasma osmolality in three vascular beds. Am J Physiol 1971;220:384-391.

15 Velasco IT, Pontieri V, Rocha Silva M Jr, Lopes OU: Hyperosmotic $\mathrm{NaCl}$ and severe hemorrhagic shock. Am J Physiol 1980;239: H664-H673.

16 Waagstein L, Haljamae H, Ricksten SE, Sahlman L: Effects of hypertonic saline on myocardial function and metabolism in nonischemic and ischemic isolated working rat hearts. Crit Care Med 1995;23:1890-1897.

17 Kreimeier U, Messmer K: Small volume resuscitation with hypertonic-hyperoncotic solutions; in Kox J, Gamble J (eds): Fluid Resuscitation. Baillière's Clinical Anaesthesiology. London, Ballière Tindall, 1988, vol 2, pp 545577.

18 Granger DN, Benoit JN, Suzuki M, Grisham MB: Leukocyte adherence to venular endothelium during ischemia-reperfusion. Am J Physiol 1989;257:G683-G688.

19 Pardi R, Inverardi L, Bender JR: Regulatory mechanisms in leukocyte adhesion: Flexible receptors for sophisticated travelers. Immunol Today 1992;13:224-230.

20 Nolte D, Bayer M, Lehr HA, Becker M, Krombach F, Kreimeier U, Messmer K: Attenuation of postischemic microvascular disturbances in striated muscle by hyperosmolar saline dextran. Am J Physiol 1992;263:H1411-H1416.

21 Groner W, Winkelman JW, Harris AG, Ince C, Bouma GJ, Messmer K, Nadeau RG: Orthogonal polarization spectral imaging: A new method for study of the microcirculation. Nat Med 1999;5:1209-1212.

22 Szabó A, Suki B, Csonka E, Eszlari E, Kucsa K, Vajda K, Kaszaki J, Boros M: Flow motion in the intestinal villi during hemorrhagic shock: A new method to characterize the microcirculatory changes. Shock 2004;21:320-328.

23 Bertuglia S, Colantuoni A: Venular oscillatory flow during hemorrhagic shock and NO inhibition in hamster cheek pouch microcirculation. Microvasc Res 1997;54:233-242.

24 Vollmar B, Preissler G, Menger MD: Hemorrhagic hypotension induces arteriolar vasomotion and intermittent capillary perfusion in rat pancreas. Am J Physiol 1994;267:H1936H1940.
25 Hudetz AG, Roman RJ, Harder DR: Spontaneous flow oscillations in the cerebral cortex during acute changes in mean arterial pressure. J Cereb Blood Flow Metab 1992;12:491-499.

26 Borgström P, Schmidt JA, Bruttig SP, Intaglietta M, Arfors KE: Slow-wave flowmotion in rabbit skeletal muscle after acute fixed-volume hemorrhage. Circ Shock 1992;36:57-61.

27 Gustafsson H, Bulow A, Nilsson H: Rhythmic contractions of isolated pressurized small arteries from rat. Acta Physiol Scand 1994;152: 145-152.

28 Rücker M, Strobel O, Vollmar B, Roesken F, Menger MD: Vasomotion in critically perfused muscle protects adjacent tissues from capillary perfusion failure. Am J Physiol 2000;279: H550-H558.

29 Appelgren L: Perfusion and diffusion in shock: A study of disturbed tissue-blood exchange in low flow states in canine skeletal muscle by a local clearance technique. Acta Physiol Scand Suppl 1972;378:1-72.

30 Funk W, Intaglietta M: Spontaneous arteriolar vasomotion; in: Progress in Applied Microcirculation. Basel, Karger, 1985, vol 3, pp 33-50.

31 Tsai AG, Intaglietta M: Evidence of flowmotion induced changes in local tissue oxygenation. Int J Microcirc Clin Exp 1993;12:75-88.

32 Lilly MP, Engeland WC, Gann DS: Adrenal medullary responses to repeated hemorrhage in conscious dogs. Am J Physiol 1986;251: R1193-R1199.

33 Gustafsson $\mathrm{H}$ : Vasomotion and underlying mechanisms in small arteries. An in vitro study of rat blood vessels. Acta Physiol Scand Suppl 1994;614:1-44.

34 Bohlen HG, Gore RW: Preparation of rat intestinal muscle and mucosa for quantitative microcirculatory studies. Microvasc Res 1976;11: 103-110.

35 Fruchterman TM, Spain DA, Wilson MA, Harris $\mathrm{PD}$, Garrison RN: Selective microvascular endothelial cell dysfunction in the small intestine following resuscitated hemorrhagic shock. Shock 1998;10:417-422.

36 Madorin WS, Martin CM, Sibbald WJ: Dopexamine attenuates flow motion in ileal mucosal arterioles in normotensive sepsis. Crit Care Med 1999;27:394-400.

37 Menger MD, Steiner D, Messmer K: Microvascular ischemia-reperfusion injury in striated muscle: significance of 'no reflow'. Am J Physiol 1992;263:H1892-H1900. 